\title{
TO WHOM IT MAY CONCERN
}

Attention: ANU Publication Officers for HERDC Data Collection

JOURNAL:

Australian Cultural History: The J ournal of the History of Culture in Australia

CHAPTER:

The Australian Democrats

I confirm that I have been affiliated with the Australian National University College of Law during the research and publication of the above chapter.

Yours sincerely

Andrew Bartlett 